\title{
The Nuclear Ring in the Barred Spiral Galaxy IC 4933
}

\author{
Stuart D. Ryder ${ }^{\mathrm{A}, \mathrm{D}}$, Samuel M. Illingworth ${ }^{\mathrm{B}}$, Robert G. Sharp ${ }^{\mathrm{A}}$, \\ and Catherine L. Farage $\mathrm{C}^{\mathrm{C}}$

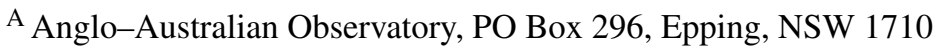 \\ ${ }^{B}$ Department of Physics \& Astronomy, University of Leicester, Leicester, LE1 7RH, UK \\ ${ }^{\text {C }}$ Research School of Astronomy and Astrophysics, Australian National University, ACT 2611 \\ D Corresponding author. Email: sdr@aao.gov.au
}

Received 2009 April 22, accepted 2009 September 2

\begin{abstract}
We present infrared imaging from IRIS2 on the Anglo-Australian Telescope that shows the barred spiral galaxy IC 4933 has not just an inner ring encircling the bar, but also a star-forming nuclear ring $1.5 \mathrm{kpc}$ in diameter. Imaging in the $u^{\prime}$ band with GMOS on Gemini South confirms that this ring is not purely an artifact due to dust. Optical and near-infrared colours alone however cannot break the degeneracy between age, extinction, and burst duration that would allow the star formation history of the ring to be unraveled. Integral field spectroscopy with the GNIRS spectrograph on Gemini South shows the equivalent width of the $\mathrm{Pa} \beta$ line to peak in the north and south quadrants of the ring, indicative of a bipolar azimuthal age gradient around the ring. The youngest star-forming regions do not appear to correspond to where we expect to find the contact points between the offset dust lanes and the nuclear ring unless the nuclear ring is oval in shape, causing the contact points to lead the bar by more than $90^{\circ}$.
\end{abstract}

Keywords: galaxies: evolution — galaxies: individual (IC 4933) — galaxies: nuclei — galaxies: starburst — stars: formation

\section{Introduction}

High spatial resolution multi-wavelength imaging has revealed a host of intriguing features close in to the nuclear regions of barred spiral galaxies, including starburst rings, dust spirals, and nested bars (e.g. Ryder \& Knapen 1999; Laine et al. 2002; Martini et al. 2003). Early numerical simulations of dynamical evolution in barred galaxies (e.g. Schwarz 1981; Athanassoula et al. 1982) appeared to link circumnuclear starburst rings with the inner Lindblad resonance (ILR), the radius at which the bar pattern speed $\Omega_{\mathrm{p}}$ is equal to $\Omega-\kappa / 2$, where $\Omega$ is the mean angular frequency, and $\kappa$ the radial epicyclic frequency. More recent hydrodynamic simulations by Regan \& Teuben (2003) have argued that star-forming nuclear rings actually result whenever a certain fraction of gas clouds find themselves on elongated orbits aligned perpendicular to, rather than parallel with, the major axis of the bar, and that furthermore these rings shrink with time.

As atomic gas in the circumnuclear regions is generally scarce, the bar (or some form of oval distortion) is thought to play a significant role in 'funneling' gas inwards via shocks to feed the competing needs of a starforming ring, and/or an Active Galactic Nucleus (AGN) component. When gas is trapped near or between resonant orbits, and is consumed in star formation, the AGN will potentially be starved and forced to go dormant. One way to overcome this 'logjam' is to create a secondary, or 'nested' bar, which can disrupt the resonances of the main bar (Englmaier \& Shlosman 2004; Heller et al. 2007). Thus, we see that nuclear rings, nested bars, and AGN are all fundamentally inter-related, and yet we have surprisingly little data on their relative frequencies (Knapen 2005), or causal connections (Laine et al. 2006).

The total sample of nuclear rings of sufficient angular size to be resolved with ground-based seeing-limited spectrographs or aperture synthesis interferometers is still quite limited (Comerón et al. 2010). Galaxies exhibiting an inner ring encircling their bar very often harbour a nuclear ring as well (Buta \& Combes 1996). The nearly face-on barred spiral galaxy IC 4933 is listed in the Catalog of Southern Ringed Galaxies (CSRG; Buta 1995) as having an inner ring of $47 \times 31$ arcsec across. Table 1 summarises some other properties of this galaxy. In this paper, we report the discovery of an apparent nuclear ring in IC 4933 from near-infrared (NIR) and optical imaging, and provide spectroscopic confirmation of its nature. In Sections 2.1 and 2.2 we describe the image acquisition and reduction procedures, and analyse the colour maps. We present follow-up NIR Integral-Field Unit (IFU) spectroscopy in Section 2.3 which enables us to reconstruct the recent star formation history. Section 3 puts this in the context of an emerging picture of sequential star formation being uncovered within nuclear rings. 
Table 1. Basic data for IC 4933

\begin{tabular}{llc}
\hline Parameter & Value & Source \\
\hline Hubble type & $\mathrm{SB}(\mathrm{rs}) \mathrm{bc}$ & 1 \\
RA $(\mathrm{J} 2000)$ & $20^{\mathrm{h}} 03^{\mathrm{m}} 29^{\mathrm{s}} .04$ & 2 \\
Dec $(\mathrm{J} 2000)$ & $-54^{\circ} 58^{\prime} 47^{\prime \prime} .8$ & 2 \\
$V_{\text {hel }}\left(\mathrm{km} \mathrm{s}^{-1}\right)$ & 4915 & 3 \\
$D(\mathrm{Mpc})$ & 66 & 4 \\
Scale $\left(\mathrm{pc} \operatorname{arcsec}^{-1}\right)$ & 313 & 4 \\
Inclination $\left(\mathrm{deg}^{-1}\right)$ & 37 & 5 \\
Extinction $A_{\mathrm{B}}(\mathrm{mag})$ & 0.199 & 6
\end{tabular}

Sources: 1) Buta 1995; 2) Skrutskie et al. 2006; 3) Meyer et al. 2004; 4) NED; 5) Lauberts \& Valentijn 1989; 6) Schlegel et al. 1998.

\section{Observations and Results}

\subsection{Infrared Imaging}

Nuclear rings are seriously under-represented in the CSRG, not only because the central regions of many galaxies are overexposed on the UK Schmidt Telescope $J$ survey films on which the CSRG is based, but also due to the camouflage effect of dust within the bar region. Imaging individual galaxies at NIR wavelengths has a number of advantages in this regard:

- The extinction due to dust at $2 \mu \mathrm{m}$ is only one-tenth that at optical wavelengths (Cardelli et al. 1989).

- The rapid evolution from blue to red supergiants after the first 5-10 Myr of a starburst (Leitherer et al. 1999) moves the peak of the spectral energy distribution into the infrared. Many of these circumnuclear star formation events are not visible at all optically in this period.

- The underlying infrared background is a much better tracer of the dominant stellar population in the bar and nuclear region, allowing a better estimate of the mass-to-light ratio, and ultimately detailed modeling of the gravitational potential which can be compared with velocity fields (Buta \& Combes 2000).

The existence of a circumnuclear ring in IC 4933 was initially suspected from the difference of $J$ and $K_{\mathrm{s}}$ images obtained with the Infrared Imager and Spectrograph 2 (IRIS2; Tinney et al. 2004) instrument on the 3.9-m Anglo-Australian Telescope (AAT) in June 2003 under marginal conditions. The imaging presented here was obtained on 2006 Sep 4 UT in photometric conditions, with seeing of $1.0 \mathrm{arcsec}$ or better. The IRIS2 detector is a $1024 \times 1024$ Rockwell HAWAII-1 HgCdTe array with a pixel scale of $0.45 \operatorname{arcsec}$ pixel $^{-1}$, resulting in an instantaneous field-of-view (FoV) of $7.7 \times 7.7 \mathrm{arcmin}$.

Although the optical extent of IC 4933 is only 30\% of the IRIS2 FoV, we took a conservative approach and acquired matching observations of adjacent blank sky to track changes in the background level and illumination pattern (see Vaduvescu \& McCall (2004) for a discussion of the merits of this strategy). Five jittered observations (20 arcsec offsets) of the target galaxy were bracketed and interleaved with six jittered observations of the sky 10 arcmin south. At each object or sky jitter position, a single $30 \mathrm{~s}$ integration was recorded (in $J$ ) or $3 \times 10 \mathrm{~s}$ integrations were averaged (for $H$ or $K_{\mathrm{S}}$ ). This pattern was repeated twice, for a total on-source exposure time of 5 min in each of the $J, H$, and $K_{\mathrm{s}}$ filters.

The data reduction was carried out using the ORAC$\mathrm{DR}^{1}$ pipeline within the STARLINK package and the CHOP_SKY_JITTER recipe. Pre-processing of all raw frames included subtraction of a matching dark frame; linearity and inter-quadrant crosstalk ${ }^{2}$ correction; and bad pixel masking. The first 6 sky frames are offset in intensity to a common modal value, then a flatfield is formed from the median value at each pixel. The 6 sky frames, and 5 object frames are flatfielded, then the modal pixel values of the two sky frames bracketing each object frame are averaged and subtracted from that object frame to account for the sky background. A correction for astrometric distortion internal to IRIS2 is applied by resampling the object images, then the actual spatial offsets between images are computed using point sources common to all images. The 5 object images are mosaiced together by applying offsets in intensity to the registered images to produce the most consistent sky value possible in the overlap regions. A new flatfield and mosaic is constructed for the second set of 6 sky and 5 object frames, then the two mosaics are in turn registered and co-added to form a master mosaic.

Point sources detected in the master mosaic have been cross-referenced against the 2MASS Point Source Cata$\log$ (Skrutskie et al. 2006) and a new astrometric solution computed, while photometry in a 10 -arcsec aperture has enabled the photometric zero-points to be determined in each filter, after transforming from the 2MASS $J H K_{\mathrm{S}}$ system to the Mauna Kea Observatories (MKO) $J H K$ system (Ryder 2007).

Figure 1 shows the $J$-band $(1.25 \mu \mathrm{m})$ image of IC 4933 on a logarithmic scale. The bar, inner ring, and two stubby spiral arms are evident. The bright compact nucleus is well-fit by a Gaussian with FWHM of 2.0 arcsec, but shows no obvious indication of unusual circumnuclear structure. Aligning and subtracting the calibrated surface brightness images to form colour index maps tells a different story. Figure 2 shows the $(J-H)$ colour of the central $18 \times 23$ arcsec region, as outlined in Figure 1. An almost perfectly circular ring 5 arcsec $(1.5 \mathrm{kpc})$ in diameter stands out by virtue of its colour; whereas the underlying stellar population in the bar and bulge has $(J-H) \sim 0.65$, the ring has $(J-H)>0.7$ and is as red as 0.8 in the northwest quadrant. The $(H-K)$ map in Figure 3 has less contrast, but confirms the existence of this feature. The western half of the ring has $(H-K)>0.43$, reaching 0.48 in the northwest quadrant, while the background colour averages 0.39 .

\footnotetext{
${ }^{1}$ http: //www. oracdr.org/.

2 http: //www. eso.org/ gfinger/hawaii_1Kx1K/ crosstalk_rock/crosstalk.html.
} 


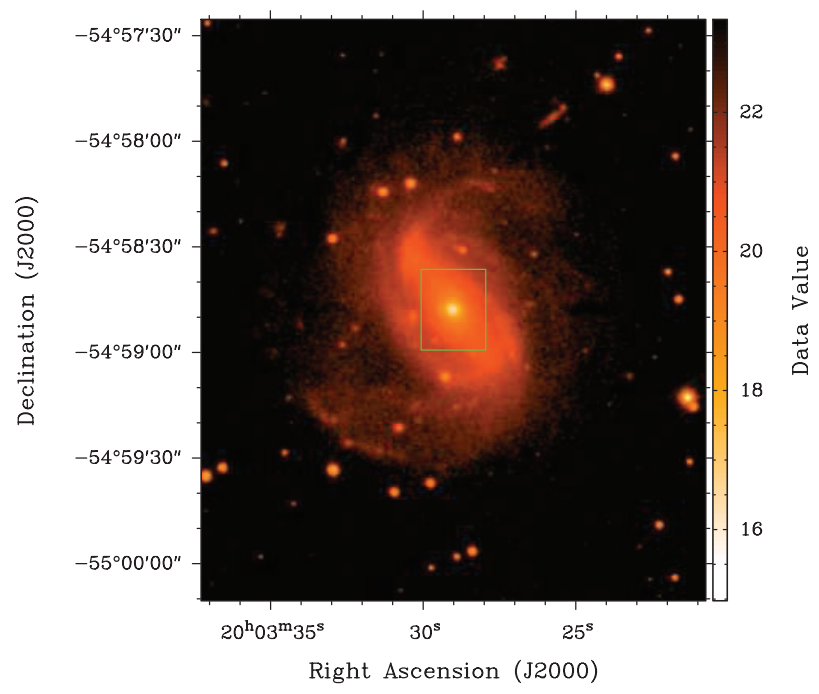

Figure 1 IRIS2 $J$-band image of IC 4933. The corresponding surface brightness in units of mag arcsec ${ }^{-2}$ is indicated by the colour bar at right. The inset box outlines the region shown in the colour index maps in Figures 2 and 3.

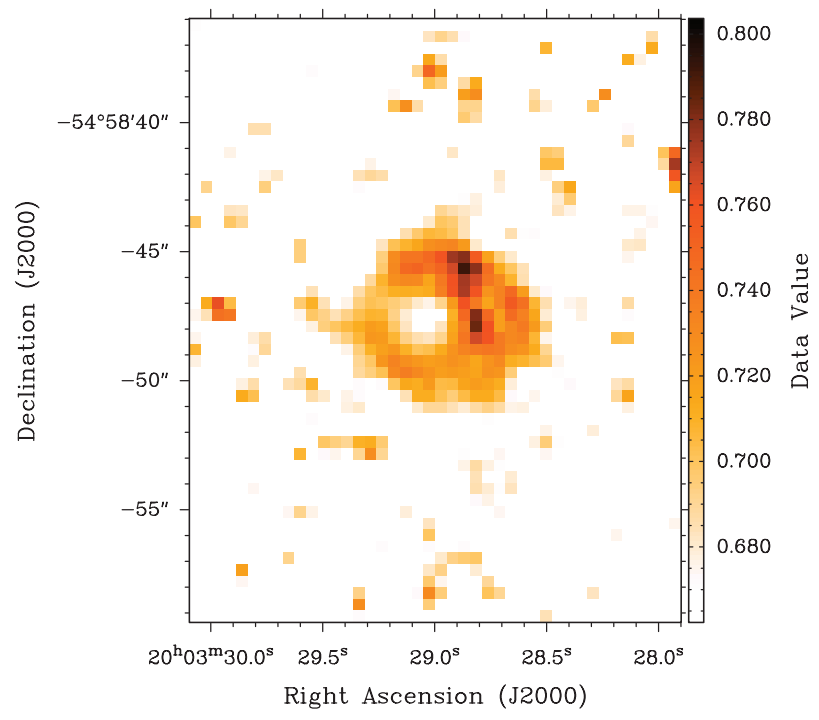

Figure 2 IRIS2 $(J-H)$ colour index map of the inner bar region of IC 4933. The colour range displayed is indicated by the scale at right.

In Figure 4 we plot the $(J-H)$ colour against the $(H-K)$ colour averaged over a $2 \times 2$ pixel area at the 4 cardinal points around the ring, as well as the mean colours of the background stellar population. The reddening vector corresponding to 2 mag of extinction in the $V$ band is also plotted. We have used the Starburst 99 models from Leitherer et al. (1999) to compute the evolutionary track through this two colour diagram of a stellar population having an Initial Mass Function with a Salpeter-like slope $(\alpha=2.35)$ between 1 and $100 \mathrm{M}_{\odot}$, and super-solar metallicity $(Z=0.04)$ typical of the bulges in Milky Waylike systems such as IC 4933. To encompass most star formation histories, Leitherer et al. (1999) consider two limiting cases: an 'Instantaneous' burst of star formation (IB), and a 'Continuous' constant rate of star formation

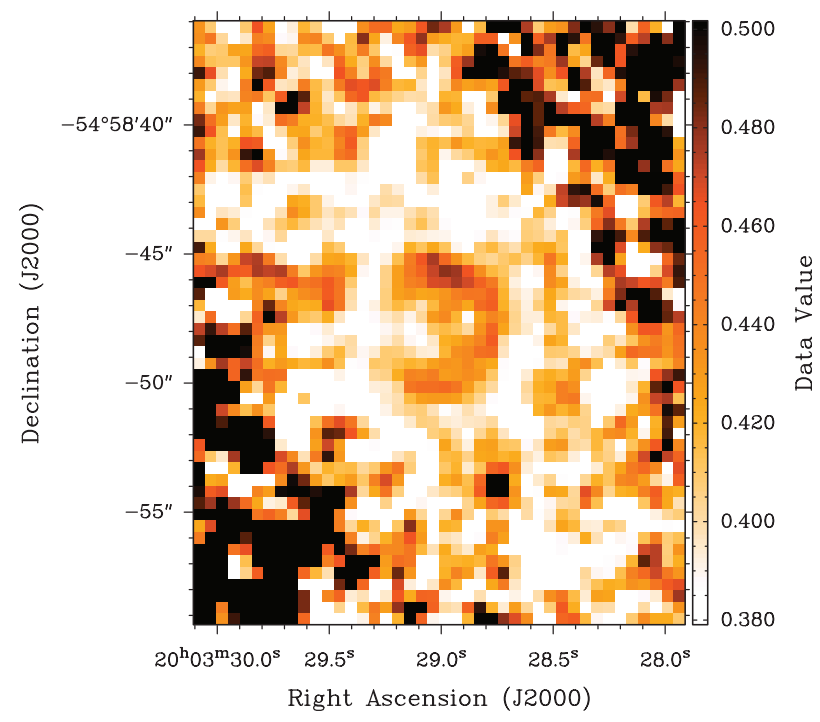

Figure 3 IRIS2 $(H-K)$ colour index map of the inner bar region of IC 4933. The colour range displayed is indicated by the scale at right.

(CSF). The evolution over $250 \mathrm{Myr}$ for these two scenarios is marked on Figure 4. Both start off with almost identical colours, then evolve rapidly bluewards for the first 57 Myr until reversed by the evolution of massive blue stars into red supergiants. The destruction of these red supergiants in core-collapse supernovae allows the colours to again evolve bluewards after $\sim 9 \mathrm{Myr}$ and $\sim 40 \mathrm{Myr}$ for the IB and CSF models, respectively, but now with $(J-H)$ being 0.5 mag redder than on the initial 'blue loop'.

At no stage do these evolutionary tracks achieve colours as red as those measured around the ring. While the colours of the background stellar population are similar to those reached by the IB model in the brief period $8-20 \mathrm{Myr}$ with almost no reddening, this is an extremely unlikely scenario as the amount of nuclear dust highlighted in Figure 5 indicates. Rather, being more like the intermediate age population from continuous star formation marked by the triangle in Figure 4, the stellar background light in the bar and nuclear region would experience $A_{\mathrm{V}} \sim 2$ mag of extinction as indicated by the reddening vector. The ring colours are consistent with an IB population at just $5 \mathrm{Myr}$ old with up to $A_{\mathrm{V}}=6$ mag of extinction, with the required reddening dropping to $0.5-1 \mathrm{mag}$ for an age of $9 \mathrm{Myr}$. The implied range of extinction from the CSF models is just $A_{\mathrm{V}}=1.5-2.5 \mathrm{mag}$, but the allowable range of ages is much greater at 10-100 Myr. It is this degeneracy between extinction, age, and even the duration of star formation that requires us to seek better diagnostics than broadband NIR colours (Section 2.3).

\subsection{Optical Imaging}

As Figure 4 indicates, an alternate scenario is that the ring is simply dust seen in silhouette, reddening and obscuring the background population by the equivalent of between 0.3 and 1.4 mag of extinction in $V$. While linear dust lanes and nuclear dust spirals are relatively frequent in spiral 


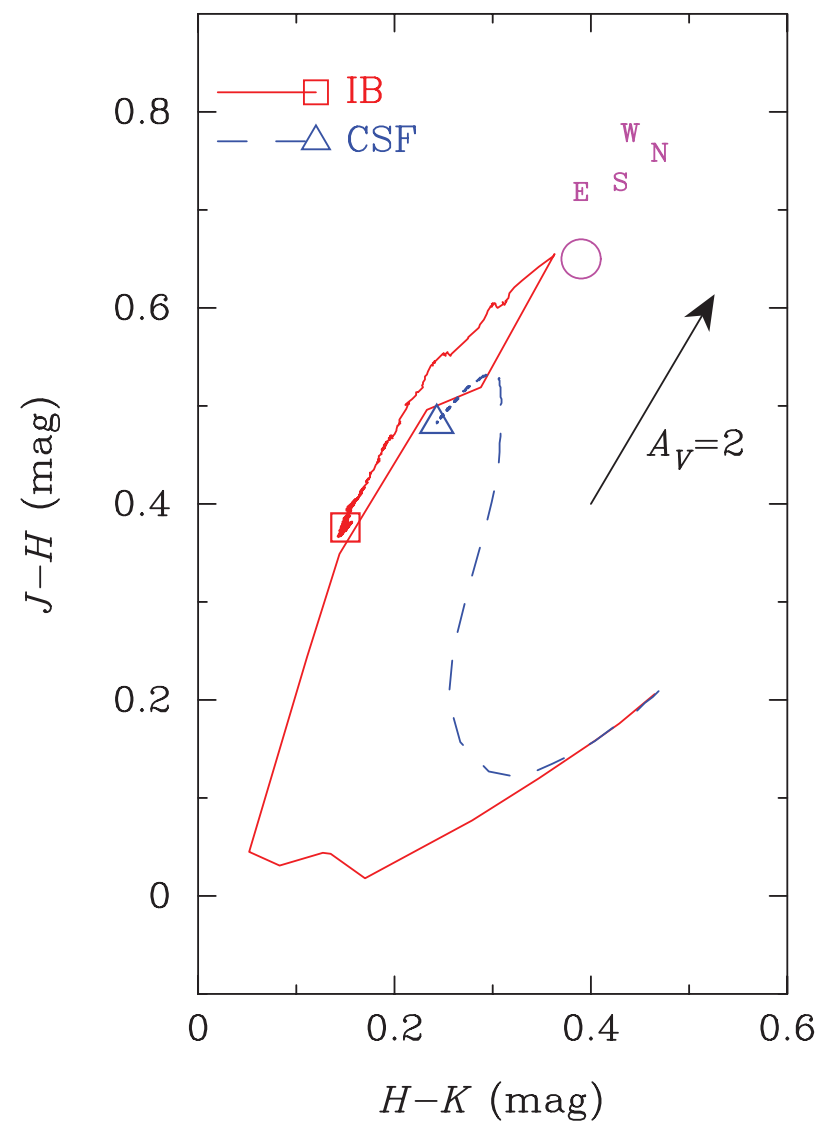

Figure 4 Two colour diagram for 4 specific locations around the circumnuclear ring in IC 4933: (N)orth, (W)est, (S)outh, and (E)ast. The large open circle indicates the spread of colours from unresolved stars in the bulge and bar. The arrow indicates the effect on the NIR colours from reddening equivalent to 2 mag of extinction in the $V$ band. The solid line is the evolutionary track of a stellar population formed from an instantaneous burst (IB) according to Leitherer et al. (1999), reaching the open square after $250 \mathrm{Myr}$. The dashed line ending at the open triangle is the evolutionary track of a stellar population undergoing continuous star formation (CSF) over $250 \mathrm{Myr}$.

galaxy nuclei (Martini et al. 2003), and compact nuclear dust disks have been seen around some AGN (Jaffe et al. 1996; van der Marel \& van den Bosch 1998), such a kiloparsec-scale coherent dust ring has not been seen elsewhere.

To test for the presence of a dust ring, we need a much larger extinction contrast than that offered by NIR colours. Images of IC 4933 in the Sloan Digital Sky Survey (SDSS) $u^{\prime}, g^{\prime}$, and $r^{\prime}$ filters were obtained with the Gemini MultiObject Spectrograph (GMOS; Hook et al. 2004) attached to the Gemini South Telescope as part of programme GS-2008A-Q-204 (PI: S. Ryder) in the 'Poor Weather' queue (seeing $>1.1$ arcsec in non-photometric conditions with $>2$ mag of atmospheric extinction) on 2008 July 7 UT. Only the $u^{\prime}$ data is presented here. Three exposures of $690 \mathrm{~s}$ each were obtained, with each exposure offset by 8 arcsec spatially to allow filling-in of the inter-CCD gaps in GMOS. On-chip binning yielded a pixel scale of $0.292 \operatorname{arcsec} \mathrm{pix}^{-1}$.

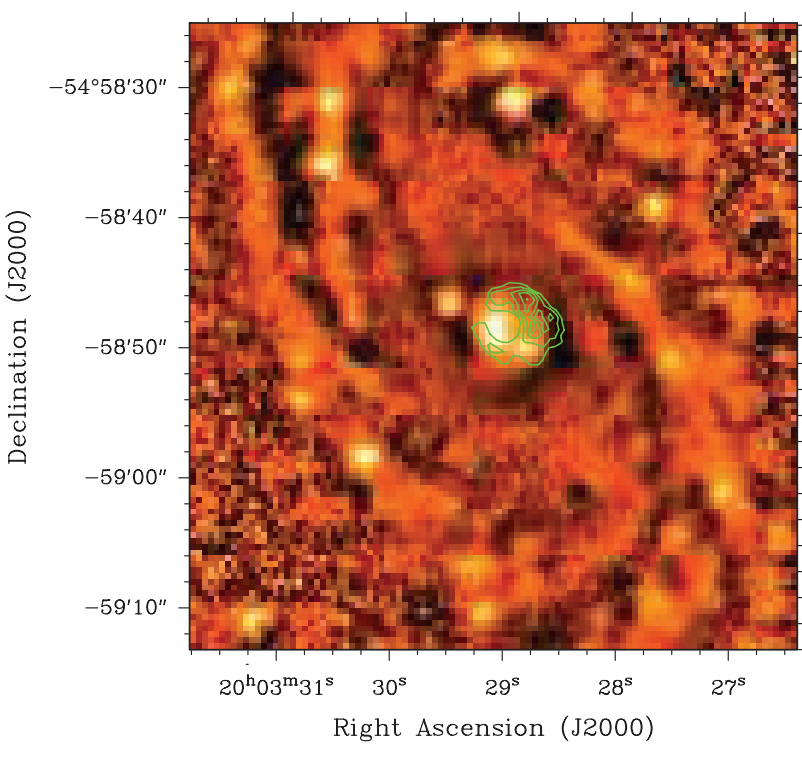

Figure 5 Contours of $(J-H)$ colour in the nucleus of IC 4933 from IRIS2, superimposed on a map of the $\left(u^{\prime}-K\right)$ colour index in bar and nuclear region from the difference of GMOS and IRIS2 images. An unsharp mask has been applied to the $\left(u^{\prime}-K\right)$ image in order to highlight the two dust lanes which trace the leading edge of the bar and encircle the nuclear ring seen in $(J-H)$. The contours correspond to $(J-H)=(0.71,0.73,0.75,0.77,0.79)$, while the bluest $\left(u^{\prime}-K\right)$ colours appear white in this figure.

The data were reduced and combined using V1.9.1 of the gemini package within $\operatorname{IRAF}^{3}$. A master bias frame (constructed by averaging with 3- $\sigma$ clipping a series of bias frames) was subtracted from all raw images in lieu of overscan fitting and subtraction. Images of the twilight sky were used to flatfield the images, then the dithered galaxy images were registered and averaged together with the imcoadd task to eliminate the inter-CCD gaps, bad pixels, and cosmic rays. No absolute flux calibration of this data is available, but for the purposes of constructing colour index maps the final image has been converted to a logarithmic scale.

This $u^{\prime}$ image has been scaled and aligned to match the IRIS2 $K$ image using the geomap and geotran tasks before the latter was subtracted from the former to produce a $\left(u^{\prime}-K\right)$ colour index map which spans a factor of 6 in wavelength. This map has been unsharp masked by subtracting off a lightly smoothed version of itself, to help accentuate any apparent dust lane structure. Figure 5 shows this difference in optical versus NIR morphology, together with a contoured representation of the ring seen in $(J-H)$. One can quite clearly see dust lanes offset either side of the bar major axis and slightly concave towards it. As shown in the simulations of Athanassoula (1992), such offset curved dust lanes mark the loci of shocks at the leading edge of a relatively fat bar. These dust lanes curl around the nucleus and encircle it, though this dust

\footnotetext{
${ }^{3}$ IRAF is distributed by the National Optical Astronomy Observatories, which are operated by the Association of Universities for Research in Astronomy, Inc., under cooperative agreement with the National Science Foundation.
} 
ring is noticeably larger $(\sim 8$ arcsec diameter $)$ than the NIR ring found in Section 2.1. Within this dust ring sits three blue 'hot spots', two of which are concentric with the NIR ring and which in fact coincide with the bluest $(J-H)$ sections of the NIR ring.

\subsection{IFU Spectroscopy}

Near-IR spectroscopy can place much tighter constraints on the ages and star-forming timescales of individual clusters (Alonso-Herrero et al. 2001; Ryder et al. 2001). The combination of multiple, independent age diagnostics (including the $\operatorname{Br} \gamma$ 2.16- $\mu \mathrm{m}$ equivalent width; the $\mathrm{CO}(2,0) 2.3-\mu \mathrm{m}$ spectral index; or the [FeII] 1.26- $\mu \mathrm{m}$ luminosity) can in principle discriminate the IB from the CSF models of Leitherer et al. (1999), as well as their relative ages. Since most of these diagnostics involve the ratio of an emission/absorption feature to their adjacent continuum emission, they are far less affected by differential extinction than the broadband colour indices in Section 2.1.

Spectroscopy of the circumnuclear ring in IC 4933 was conducted in queue mode with the Gemini NearInfraRed Spectrograph (GNIRS; Elias et al. 2006) with its Integral Field Unit (IFU; Allington-Smith et al. 2006) attached to the Gemini-South Telescope for program GS2005A-Q-25 (PI: S. Ryder). The GNIRS IFU slices a $4.8 \times 3.15$ arcsec FoV into 21 slices 0.15 arcsec wide by 4.8 arcsec long and reformats it into a single 'long slit', with each of the 21 slices projecting to 320.15 arcsec pixels on the $1024 \times 1024$ Aladdin InSb array. The 31.7 lines $\mathrm{mm}^{-1}$ grating and short camera disperse the stacked slices at a resolving power $\lambda / \Delta \lambda \sim 1700$, with separate grating settings spanning much of the $J$ band $(1.06-1.45 \mu \mathrm{m})$ and the $K$ band $(1.90-2.56 \mu \mathrm{m})$.

As the ring just overfills the IFU FoV, four adjacent pointings were observed, effectively covering each quadrant of the ring in turn. Each pointing was observed in 4 integrations of $250 \mathrm{~s}$, with 32 reads per integration delivering a read noise of 7 electrons. Each of the 4 integrations was dithered by $\pm 0.3 \operatorname{arcsec}$ ( 2 pixels) in both axes to compensate for reduced throughput in the two outer and one of the central slices, and to provide 2 pixels of overlap between pointings. Furthermore, each integration was interleaved with equivalent integrations of blank sky 1 arcmin east, to enable removal of the telluric $\mathrm{OH}$ and thermal emission. Observations of the F0 V stars HD 176193 and HD 200767, nodding between each half of the IFU, were made at comparable airmass to the IC 4933 observations to correct for telluric absorption. Observations were conducted over 5 nights in March and April 2005 when conditions were photometric, in seeing of 0.8-1.0 arcsec. Spectra of an Argon arc lamp provided the wavelength calibration, while observations of a $1100 \mathrm{~K}$ 'grey body' or quartz halogen lamp facilitated the correction for pixel-to-pixel sensitivity variation.

The IFU data has been reduced using the gnirs package within IRAF. Images taken in 'Bright Objects' read mode (i.e. telluric standards, flatfields, and arcs) were found to exhibit a ripple pattern noise along each column, having a period of 17 pixels and amplitude $\sim 260$ electrons, well in excess of the 38 electrons read noise expected in this mode. We have been able to remove this ripple pattern by median filtering the unexposed columns, then subtracting this from every column in the image. The 32 columns corresponding to each of the 21 slices are extracted into individual image planes. Each of the 21 object slices has the matching nodded object or blank sky slice subtracted from it, and is then divided by the normalised, smoothed flatfield lamp slice. Tracing of an artificial 'pinhole' spectrum allows each slice plane to be straightened in the spatial direction, while interactive arc line identification and fitting is used to rebin each slice on a linear wavelength scale.

Subsequent processing has been carried out using purpose-written IDL routines. For the IC 4933 observations the 21 slices are stacked into a 3D datacube of $32 \times 21$ spatial pixels $\times 1022$ spectral elements. The 4 datacubes from each of the dithered pointings covering each quadrant are registered and combined into one datacube per quadrant. The 4 quadrant cubes have then been mosaiced into a master datacube covering the entire ring and its surroundings.

For the stellar observations, columns containing high signal-to-noise spectra are extracted and summed; Gaussian profiles are fitted to the hydrogen recombination lines (e.g. $\mathrm{Pa} \beta$ at $1.28 \mu \mathrm{m}, \mathrm{Br} \gamma$ at $2.16 \mu \mathrm{m}$ ) then used to fill in this absorption; and finally they are divided by the Planck function appropriate to a blackbody of the same temperature as the star. This yields response spectra which are divided into each spectrum of the $J$-band and $K$-band master datacubes to compensate for variations in atmospheric transmission with wavelength.

Inspection of the datacubes does indicate the presence of extended line emission at the redshifted wavelengths of $\mathrm{Pa} \beta, \mathrm{Br} \gamma$, and $\mathrm{H}_{2}$ 1-0 S(1). However, the net signalto-noise in the $K$-band master datacube is significantly poorer than the $J$ band, and inadequate for our purposes. We therefore have to rely solely on the $J$-band datacube for our analysis. The equivalent width of the $\mathrm{Pa} \beta$ emission line has been measured in the datacube by integrating the flux within the line and dividing by the interpolated continuum level. Figure 6 shows a map of the $\mathrm{Pa} \beta$ equivalent width after binning $\times 3$ to match the pixel scale of IRIS2, overlaid with contours of the $(J-H)$ colour from Figure 2.

By contrast with the broad-band colour maps, the $\operatorname{Pa} \beta$ map shows a clear bifold symmetry, with the equivalent width reaching a maximum of $14 \pm 2 \AA$ almost due North and South around the ring, and a minimum of $5.5 \pm 0.5 \AA$ in the East and West. Figure 7 shows the evolution of $\mathrm{Pa} \beta$ equivalent width with time for the IB and CSF Starburst99 models of Section 2.1.

The IB model reaches the $\mathrm{Pa} \beta$ levels of the North/South quadrants $6.6 \mathrm{Myr}$ after the onset of the burst, and those of the East/West quadrants a further 0.3 Myr later. This is perfectly consistent with the IB colour evolution described by Figure 4, with azimuthal variations in 


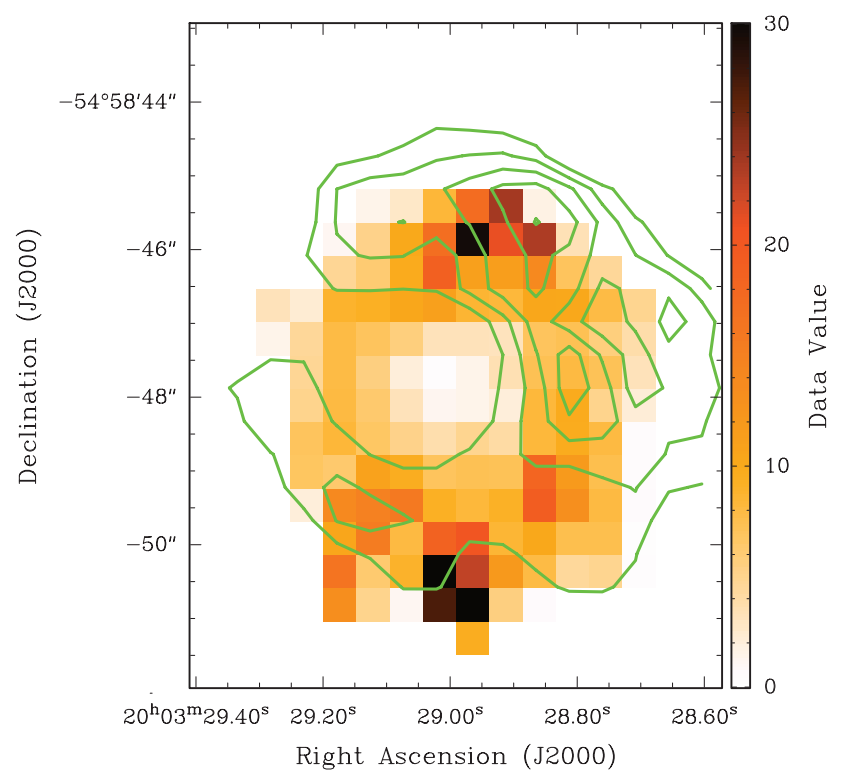

Figure 6 Contours of $(J-H)$ colour in the nucleus of IC 4933 from IRIS2, superimposed on a map of the $\mathrm{Pa} \beta$ equivalent width from GNIRS IFU spectroscopy. The contours correspond to $(J-H)=(0.71,0.73,0.75,0.77,0.79)$, while the equivalent width in units of $\AA$ is indicated by the scale at right.

extinction accounting for the morphological differences between the nuclear ring as seen in $(J-H)$ and in $\mathrm{Pa} \beta$ (Figure 6). Indeed, the extinctions inferred for each quadrant from their derived ages and location in Figure 4 are found to range from $A_{\mathrm{V}} \sim 2$ mag in the E quadrant, up to 3.5 mag in the $\mathrm{N}$ quadrant.

Although this plot covers only the first $10 \mathrm{Myr}$, even $1 \mathrm{Gyr}$ after the onset of CSF the $\mathrm{Pa} \beta$ equivalent width is still $50 \%$ higher than the peak values observed anywhere around the ring. While it is conceivable that the observed $\mathrm{Pa} \beta$ equivalent widths are underestimated due to inadequate allowance for an older background stellar population contaminating the continuum fluxes, the very gradual decline in $\mathrm{Pa} \beta$ equivalent width over time in the CSF model would make the East/West quadrants at least 0.5 Gyr older than the North/South quadrants. We can conceive of no scenario under which two populations of such differing ages could remain spatially distinct for so long in such a compact, but kinematically active region and therefore discount purely continuous star formation in the circumnuclear ring of IC 4933 as playing any significant role.

\section{Discussion}

The IB and CSF Starburst99 models represent the two extremes of star formation, and as stellar population model fits to optical line indices of nuclear rings by Sarzi et al. (2007) has indicated, the truth is more likely to be a series of multiple, episodic bursts superimposed on an old underlying bulge population. Nevertheless, the age differences around the ring are unmistakable. IC 4933 thus joins a small, but growing list of galaxies whose nuclear rings

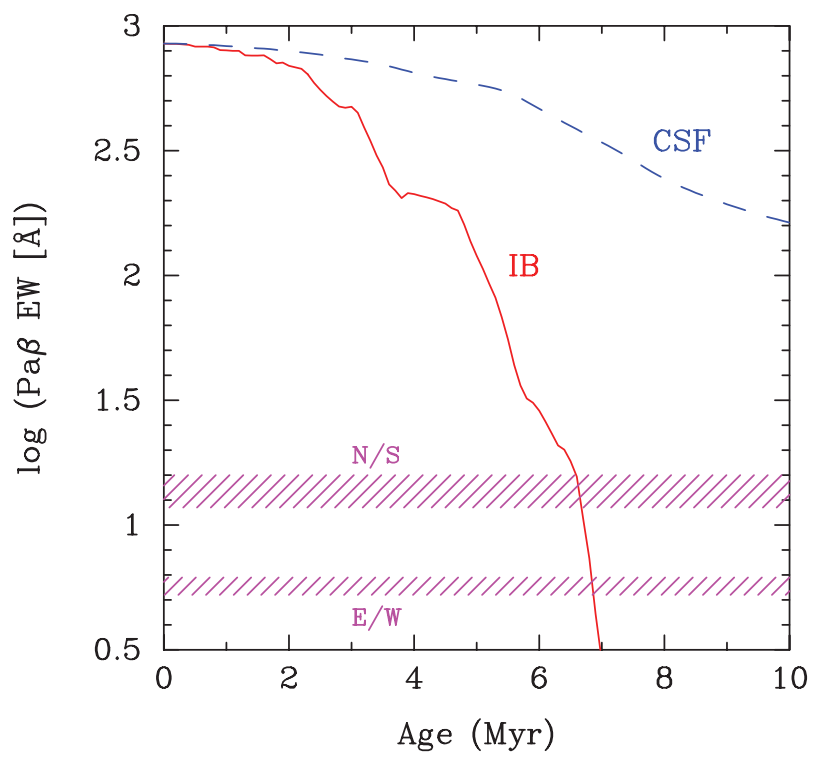

Figure 7 The evolution with time of the $\mathrm{Pa} \beta$ equivalent width for the same Starburst99 IB and CSF models as in Figure 4. The upper and lower shaded regions mark the observed range of equivalent widths within the North/South, and East/West quadrants respectively, of the IC 4933 nuclear ring.

exhibit evidence of a clear bipolar azimuthal age gradient such as M100 (Ryder et al. 2001; Allard et al. 2006), NGC 1343, and NGC 1530 (Mazzuca et al. 2008). Many more galaxies show some form of azimuthal age gradient at least, like that in M83 (Puxley et al. 1997; Harris et al. 2001; Knapen et al. 2010), including $60 \%$ of the sample of Böker et al. (2008) observed in the NIR with the SINFONI IFU on the VLT, and $40 \%$ of the sample of Mazzuca et al. (2008) observed in $\mathrm{H} \alpha$.

Mazzuca et al. (2008) found no clear relationship between the presence or absence of an age gradient, and the morphology of either the ring or the host galaxy. They did note that the rings in their sample that show no clear age gradient are also among the oldest ( $>10 \mathrm{Myr}$ ) starforming rings. Thus it may be that with the passage of time and several complete orbits of the aging clusters around the ring that a well-organised, sequential star formation pattern may simply be smoothed over (Böker et al. 2008). We do know that the starburst in IC 4933 is comparatively young and of short duration, which may partly account for why it still has such a well-defined bipolar age gradient. A weak or intermittent gas inflow rate from the bar into the ring may also yield a more stochastic age distribution of clusters around the ring, particularly if star formation only occurs above some critical threshold density (Elmegreen 1997; Böker et al. 2008; Mazzuca et al. 2008).

Mazzuca et al. (2008) plotted the angular offset between the bar major axis position angle and the azimuth of the youngest Hir region around each half of their nuclear rings. They found a broad distribution with an apparent peak around $90^{\circ}$, suggesting that soon after inflowing gas reaches the 'contact points' between the offset dust lanes and the nuclear ring (commonly found perpendicular to the bar major axis) it is converted to stars. We have used the 
ellipse surface photometry task within IRAF to derive the bar position angle. Over radial distances between 10 and 18 arcsec where the bar dominates, the position angle is $(32 \pm 2)^{\circ}$. The two $\mathrm{Pa} \beta$ peaks in Figure 6 have position angles of $182^{\circ}$ and $350^{\circ}$, so are displaced some $30-40^{\circ}$ from the bar position angle. The spatial resolution of Figure 5 is insufficient to discern if the contact points in IC 4933 are indeed at $90^{\circ}$ to the bar major axis, or could in fact be much closer to these youngest Hir regions.

The GNIRS $\mathrm{Pa} \beta$ datacube allows us to extract a crude velocity field which yields a deprojected rotation velocity around the ring of $220 \mathrm{~km} \mathrm{~s}^{-1}$ with the northeast side of the disk approaching us. Assuming a bar pattern speed in the region of $25 \mathrm{~km} \mathrm{~s}^{-1} \mathrm{kpc}^{-1}$ (Ryder et al. 1996), newlyformed clusters would then drift away from the contact point at $\sim 200 \mathrm{~km} \mathrm{~s}^{-1}$. At that velocity, one complete orbit of the ring takes just $23 \mathrm{Myr}$. So we should perhaps not be too surprised to find relatively young clusters so far from the inferred current location of the contact points, where star formation could be happening right now but will not become apparent even in the NIR for a few Myr.

Alternatively, as shown by the simulations of Heller \& Shlosman (2006), an oval (rather than circular) nuclear ring can result in contact points which are not perpendicular to the bar major axis. It is notable that the nuclear ring seen in the colour maps and in $\mathrm{Pa} \beta$ is quite circular, whereas IC 4933 appears to be inclined to the line-of-sight by $37^{\circ}$ (Lauberts \& Valentijn 1989). Thus, foreshortening of an eccentric nuclear ring could result in it appearing circular in the plane of the sky, and it may in fact be that the contact points of the dust lanes with the ring do coincide with the youngest Hir regions.

Interestingly, the blue 'hot spots' in the $\left(u^{\prime}-K\right)$ image (Figure 5) also show large spatial offsets from the $\mathrm{Pa} \beta$ equivalent width peaks. The Starburst 99 IB model in Figures 4 and 7 predicts (in the absence of any reddening) that $(U-K)$ becomes bluer by $1 \mathrm{mag}$ in the first $4-5 \mathrm{Myr}$, then reddens rapidly by 4 mag in the next 3 Myr and stays within $1 \mathrm{mag}$ of this thereafter. For these hot spots to appear as blue as they do, in the presence of dust, would require that they be no older than $5 \mathrm{Myr}$. This is inconsistent with the chronology derived from $\mathrm{Pa} \beta$ equivalent widths, which are far less susceptible to reddening, and again highlights the risk in relying solely on broadband colour measurements for deriving star formation histories on short $(<10 \mathrm{Myr})$ timescales.

\section{Conclusions}

Motivated by the fact that barred spiral galaxies known to possess large-scale inner rings are more likely to host nuclear rings as well, we obtained $J, H$, and $K_{\mathrm{s}}$ imaging of the nearby SB(rs)bc galaxy IC 4933 with IRIS2 on the AAT, and $u^{\prime}$ imaging with GMOS on Gemini South. Although the reduced images show only the bulge, bar, and arms, an almost circular ring $5 \operatorname{arcsec}(1.5 \mathrm{kpc})$ in diameter is seen in the colour index maps. Comparison of the ring NIR colours with evolutionary tracks from Starburst99 models is unable to distinguish between a burst population just $5 \mathrm{Myr}$ old, and less-reddened continuous star formation over the past 10-100 Myr.

The equivalent width of the $\operatorname{Pa} \beta$ line is much less susceptible to reddening effects. We have mosaiced four pointings of the GNIRS Integral Field Unit in order to map the $\operatorname{Pa} \beta$ equivalent width around the ring. We find clear peaks in the equivalent width in the northern and southern sectors which are most easily accounted for by short-lived bursts of star formation 6.6 Myr ago rather than by continuous star formation, with the eastern and western sectors appearing to be $0.3 \mathrm{Myr}$ older. IC 4933 is the fourth galaxy seen to have evidence of such a well-defined bipolar azimuthal age gradient. Although the actual location of the contact points of the offset dust lanes in the bar with the nuclear ring cannot be clearly discerned in our images, the significant azimuthal offset between the youngest HII regions in the ring and the bar major/minor axes are consistent with this nuclear ring being intrinsically oval in shape but fortuitously appearing circular from our perspective. Ongoing and future NIR imaging surveys such as those conducted with UKIRT and VISTA can be expected to yield many more such examples of nuclear rings, but only a comprehensive NIR integral field spectroscopic followup can unambiguously define the star formation history around these rings.

\section{Acknowledgments}

Based in part on observations obtained at the Gemini Observatory, which is operated by the Association of Universities for Research in Astronomy, Inc., under a cooperative agreement with the NSF on behalf of the Gemini partnership: the National Science Foundation (United States), the Science and Technology Facilities Council (United Kingdom), the National Research Council (Canada), CONICYT (Chile), the Australian Research Council (Australia), Ministério da Ciência e Tecnologia (Brazil) and Ministerio de Ciencia, Tecnología e Innovación Productiva (Argentina). This publication makes use of data products from the Two Micron All Sky Survey (a joint project of the University of Massachusetts and the Infrared Processing and Analysis Center/California Institute of Technology), as well as the NASA/IPAC Extragalactic Database (NED, which is operated by the Jet Propulsion Laboratory, California Institute of Technology), funded by the National Aeronautics and Space Administration and the National Science Foundation. We thank the referee for some helpful suggestions.

\section{References}

Allard, E. L., Knapen, J. H., Peletier, R. F. \& Sarzi, M., 2006, MNRAS, 371, 1087

Allington-Smith, J. R., Content, R., Dubbeldam, C. M., Robertson, D. J. \& Preuss, W., 2006, MNRAS, 371, 380

Alonso-Herrero, A., Ryder, S. D. \& Knapen, J. H., 2001, MNRAS, 322,757

Athanassoula, E., 1992, MNRAS, 259, 345

Athanassoula, E., Bosma, A., Creze, M. \& Schwarz, M. P., 1982, A\&A, 107, 101 
Böker, T., Falcón-Barroso, J., Schinnerer, E., Knapen, J. H. \& Ryder, S., 2008, AJ, 135, 479

Buta, R. J., 1995, ApJS, 96, 39

Buta, R. \& Combes, F., 1996, FCPh, 17, 95

Buta, R. \& Combes, F., 2000, in ASPC 197, Dynamics of Galaxies: from the Early Universe to the Present, Eds. Combes, F., Mamon, G. A. \& Charmandaris, V. (San Francisco: ASP), 11

Cardelli, J. A., Clayton, G. C. \& Mathis, J. S., 1989, ApJ, 345, 245

Comerón, S., Knapen, J. H., Beckman, J. E., Laurikainen, E., Salo, H., Martínez-Valpuesta, I. \& Buta, R. J., 2010, MNRAS, in press (arXiv:0908.0272)

Elias, J. H., Rodgers, B., Joyce, R. R., Lazo, M., Doppmann, G., Winge, C. \& Rodriguez-Ardila, A., 2006, SPIE, 6269, 36

Elmegreen, B. G., 1997, RMxAC, 6, 165

Englmaier, P. \& Shlosman, I., 2004, ApJ, 617, L115

Harris, J., Calzetti, D., Gallagher, J. S., Conselice, C. J. \& Smith, D. A., 2001, AJ, 122, 3046

Heller, C. H. \& Shlosman, I., 1996, ApJ, 471, 143

Heller, C. H., Shlosman, I. \& Athanassoula, E., 2007, ApJ, 657, L65

Hook, I., Jørgensen, I., Allington-Smith, J. R., Davies, R. L., Metcalfe, N., Murowinski, R. G. \& Crampton, D., 2004, PASP, 116,425

Jaffe, W., Ford, H., Ferrarese, L., van den Bosch, F. \& O'Connell, R. W., 1996, ApJ, 460, 214

Knapen, J. H., 2005, A\&A, 429, 141

Knapen, J. H., Sharp, R. G., Ryder, S. D., Falcón-Barroso, J., Fathi, K. \& Gutiérrez, L., 2010, MNRAS, submitted

Laine, S., Kotilainen, J. K., Reunanen, J., Ryder, S. D. \& Beck, R., 2006, AJ, 131, 701
Laine, S., Shlosman, I., Knapen, J. H. \& Peletier, R. F., 2002, ApJ, 567,97

Lauberts, A. \& Valentijn, E. A., 1989, The Surface Photometry Catalogue of the ESO-Uppsala Galaxies (Garching: ESO)

Leitherer, C. et al., 1999, ApJS, 123, 3

Martini, P., Regan, M. W., Mulchaey, J. S. \& Pogge, R. W., 2003, ApJS, 146, 353

Mazzuca, L. M., Knapen, J. H., Veilleux, S. \& Regan, M. W., 2008, ApJS, 174, 337

Meyer, M. J. et al., 2004, MNRAS, 350, 1195

Puxley, P. J., Doyon, R. \& Ward, M. J., 1997, ApJ, 476, 120

Regan, M. W. \& Teuben, P., 2003, ApJ, 582, 723

Ryder, S. D., 2007, AAO Newsletter, 111, 25

Ryder, S. D., Buta, R. J., Toledo, H., Shukla, H., Staveley-Smith, L. \& Walsh, W., 1996, ApJ, 460, 665

Ryder, S. D. \& Knapen, J. H., 1999, MNRAS, 302, L7

Ryder, S. D., Knapen, J. H. \& Takamiya, M., 2001, MNRAS, 323,663

Sarzi, M., Allard, E. L., Knapen, J. H. \& Mazzuca, L. M., 2007, MNRAS, 380, 949

Schwarz, M. P., 1981, ApJ, 247, 77

Schlegel, D. J., Finkbeiner, D. A. \& Davis, M., 1998, 500, 525

Skrutskie, M. F. et al., 2006, AJ, 131, 998, 1163

Tinney, C. G. et al., 2004, SPIE, 5492, 998

Vaduvescu, O. \& McCall, M. L., 2004, PASP, 116, 640

van der Marel, R. P. \& van den Bosch, F. C., 1998, AJ, 116, 2220 